\title{
Research on Skin Friction Drag Reduction by Vibrant Wall*
}

\author{
Kazuo YANAGIMOTO**, Satoru YAMAMOTO**, Naoki HAMAMOTO** \\ and Hiromitsu KAWAZOE*** \\ **Vehicle Function Testing Dept., Development Engineering Office, Mitsubishi Motors Corporation, \\ 1 Nakashinkiri, Hashime-cho, Okazaki, Aichi 444-8501, Japan \\ E-mail: kazuo.yanagimoto@mitsubishi-motors.com

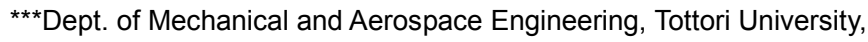 \\ 4-101 Koyama-Minami, Tottori 680-8552, Japan \\ E-mail: kawazoe@mech.tottori-u.ac.jp
}

\begin{abstract}
To reduce aerodynamic drag, reduction of skin friction is required. Vibrant flexible wall has been proposed to reduce skin friction drag for a long time. However, there are many problems for the flexible wall to get practical durability, easy maintenance and reasonable cost. On the other hand, vibrant solid wall, which is much adequate for a car body, has been rarely proposed. In this research, mechanism of drag reduction by a vibrating solid wall is considered from the velocity distribution near the wall surface. Reduction of skin friction drag on a vibrant wall is mainly caused by decrease of Reynolds shear stress, and the effect of shear stress from velocity gradient near the wall is small. The optimum condition of vibration for drag reduction is also discussed in this paper.
\end{abstract}

Key words: Turbulent Flow, Drag Reduction, Skin Friction

\section{Introduction}

For energy saving and protection of the earth environment, aerodynamic drag reduction of a car, which is effective to improve the fuel economy, has become very important. About $80 \sim 90 \%$ of the aerodynamic drag acting on a car body is the pressure drag which relates to a body shape, and the rest is the skin friction drag. Although to optimize a car shape aerodynamically makes possible to reduce its pressure drag, it is very difficult to lay out every components optimally such as engine, tires, baggage, passengers and others in order to contribute to make an aerodynamically ideal shape. Therefore, the reduction of the friction drag becomes important to decrease the aerodynamic drag more.

Previous researches show the vibrating compliant wall has a possibility to reduce friction $\mathrm{drag}^{(1),(2)}$. Recent improvement of measurement technology and numerical simulation technique suggest that to weaken an unstable T-S wave, which is induced before the transition to turbulent flow, can reduce friction $\operatorname{drag}^{(3)}$. However, the drag reduction mechanism of skin friction has not been clarified accurately.

Because of a difficulty to select proper material and from the view point of durability and maintenance, adopting a compliant wall to a car body has many problems. On the other hand, there are few results about solid vibrant wall, which is considered to be more suitable for a car body.

In this research, a passive vibrating phenomenon on a compliant wall was investigated, and the feasibility study to reproduce the same mechanism as a compliant wall by vibrating a solid wall has been conducted. The mechanism of the drag reduction was considered to be

[DOI: 10.1299/fsst.6.625]

Copyright $\odot 2011$ by JSME 
based on a friction difference between a compliant/vibrant wall and a static wall, which was deduced from the measured results of the velocity distribution near the wall surface. In addition, the optimum vibrating condition for the drag reduction is also proposed.

\section{Experimental Methods}

The small-scale wind tunnel of Mitsubishi Motors Corporation was used for the experiment. Test section of the wind tunnel has the rectangular shape with $1400 \mathrm{~mm}$ width and $900 \mathrm{~mm}$ height, and its length is $2500 \mathrm{~mm}$. Turbulence intensity of its free stream is less than $0.5 \%$. A 6 -component force balance is installed to measure aerodynamic forces. The resolution of this balance is $0.01 \mathrm{~N}$.

The feature and its size of the basic model used for this experiment are indicated in Fig. 1 and Table 1, respectively. On the upper side of the basic model, a compliant or a vibrant wall with $300 \mathrm{~mm}$ width and $800 \mathrm{~mm}$ length was equipped. And this model had four legs which were connected to 6-component force balance in the wind tunnel. Here, the coordinate was set as shown in Fig. 1.

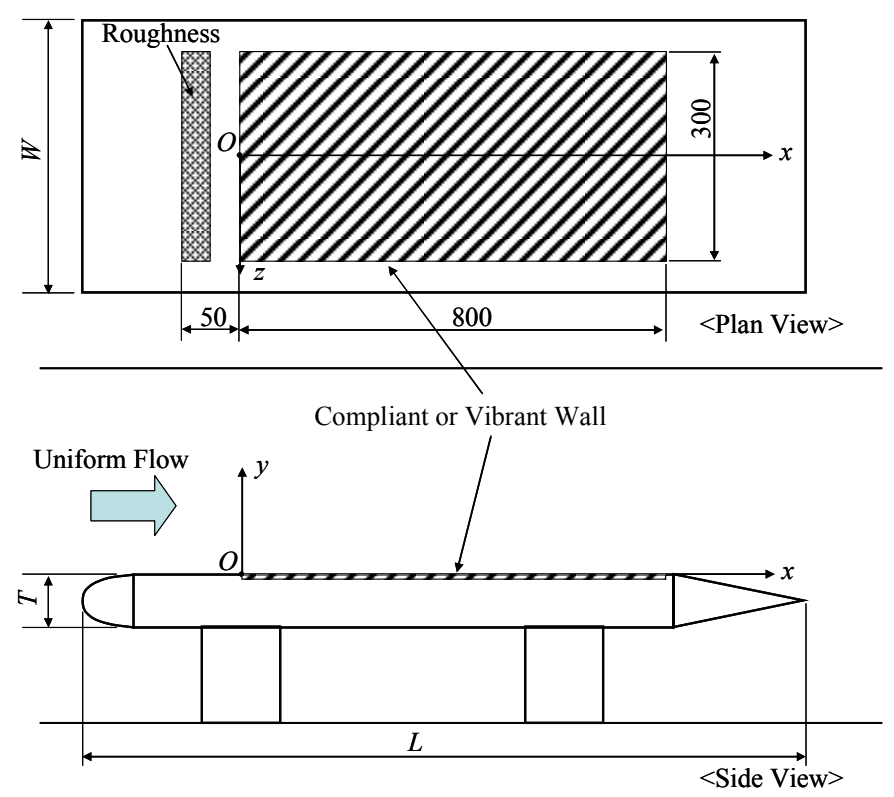

Fig. 1 Schematics of a basic model

Table 1 Size of a basic model

\begin{tabular}{|l|r|r|r|}
\hline & Length $L$ & Width $W$ & [Unit: $m$ ] $]$ \\
\hline \hline Compliant wall & 1040 & 430 & 30 \\
\hline Vibrant wall & 1370 & 350 & 100 \\
\hline
\end{tabular}

The surface of the compliant wall was rubber made, and a resin part with uneven shape was placed under the rubber surface as shown in Fig. 2. The dents of the uneven part were filled with air. Then the compliant wall could be vibrated passively. On the other hand, the vibrant wall was made from a steel plate with the thickness of $1 \mathrm{~mm}$, and oscillator was set $50 \mathrm{~mm}$ downstream from the leading edge of the vibrant wall (Fig. 3). The mini-shaker which was electro-dynamic type with a permanent field magnet (B\&K Type 4810) was used as the oscillator. It was driven by amplified sine waves generated by the function generator and the amplifier. The frequency and the amplitude of the oscillation were controlled by the sine wavelength and the gain of the amplifier, respectively. They were checked by the laser vibrometer. The steel plate was vibrated at one point by the oscillator, then transverse wave 
was spread toward each edge of a vibrant wall. In here, all edges were free and surrounding clearance was sealed with very thin tape.

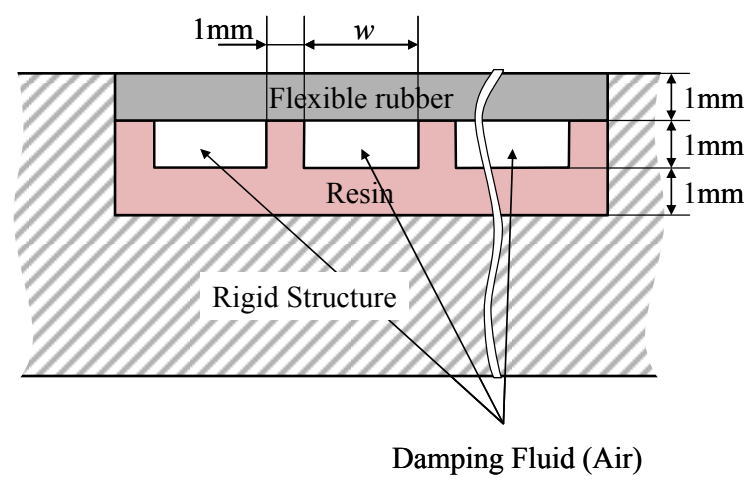

Fig. 2 Structure of the compliant wall

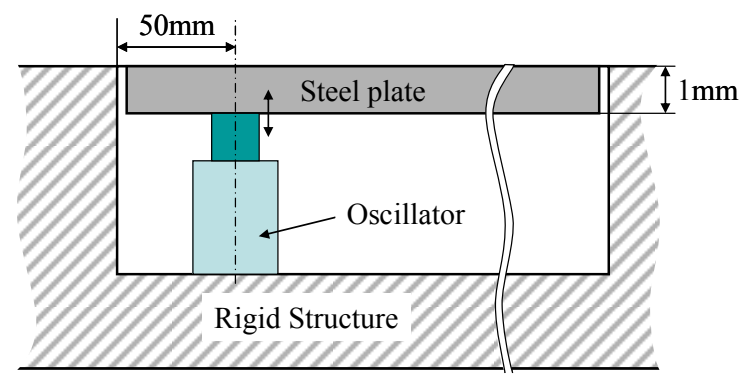

Fig. 3 Structure of the vibrant wall

To get a turbulent boundary layer on the compliant or the vibrant wall, a sand paper (\#120) was placed at $50 \mathrm{~mm}$ upstream of the leading edge of the wall as a roughness. The uniform flow velocity was $U_{\infty}=27 \mathrm{~m} / \mathrm{s}$, and Reynolds number based on the total length of the model was $2.5 \times 10^{6}$.

To catch a different amount of the friction drag between each surface and to analyze the mechanism of drag reduction by the compliant and the vibrant walls, velocity profiles and velocity fluctuations in the each boundary layers were measured. In addition, the difference of the aerodynamic drag among the compliant/vibrant wall and the solid wall was also deduced from the pressure loss in the wake of the model and skin friction at the wall.

To measure a velocity fluctuation in the boundary layer, a split film probe (Kanomax model 1288), which could measure a flow direction and a velocity fluctuation, was used. The diameter and length of its sensor were $150 \mu \mathrm{m}$ and $3.8 \mathrm{~mm}$ respectively. And the constant temperature anemometer unit (Kanomax model 1010) and the linealizer unit (Kanomax model 1013) were used for this measurement. The split film probe is made of a quartz cylinder coated with two thin platinum films, and they are separated by two gaps (split lines) along the axis of the cylinder (Fig. 4). This probe could measure unsteady velocity on the plane normal to the probe cylinder. From the measurement results, the averaged velocity and the velocity fluctuation along the $x$ - and the $y$-axes were calculated. 


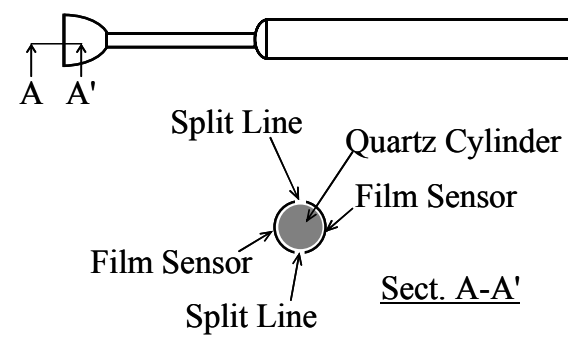

Fig. 4 Split film probe

The probe was traversed along the $y$-axis with the interval of $0.1 \mathrm{~mm}$ by the traverse unit using a micrometer (Fig. 5), and it enables to measure detailed velocity profile in the boundary layer. Shear stress $\tau$ was calculated from the following equation with measured velocity profile and velocity fluctuation in the boundary layer under the boundary layer approximation.

$$
\tau=\mu \frac{\partial u}{\partial y}-\rho \overline{u^{\prime} v^{\prime}}
$$

where, $\mu, u, \rho, u^{\prime}$ and $v^{\prime}$ are viscosity, velocity in the $x$ direction, density, velocity fluctuations in the $x, y$ directions, respectively.
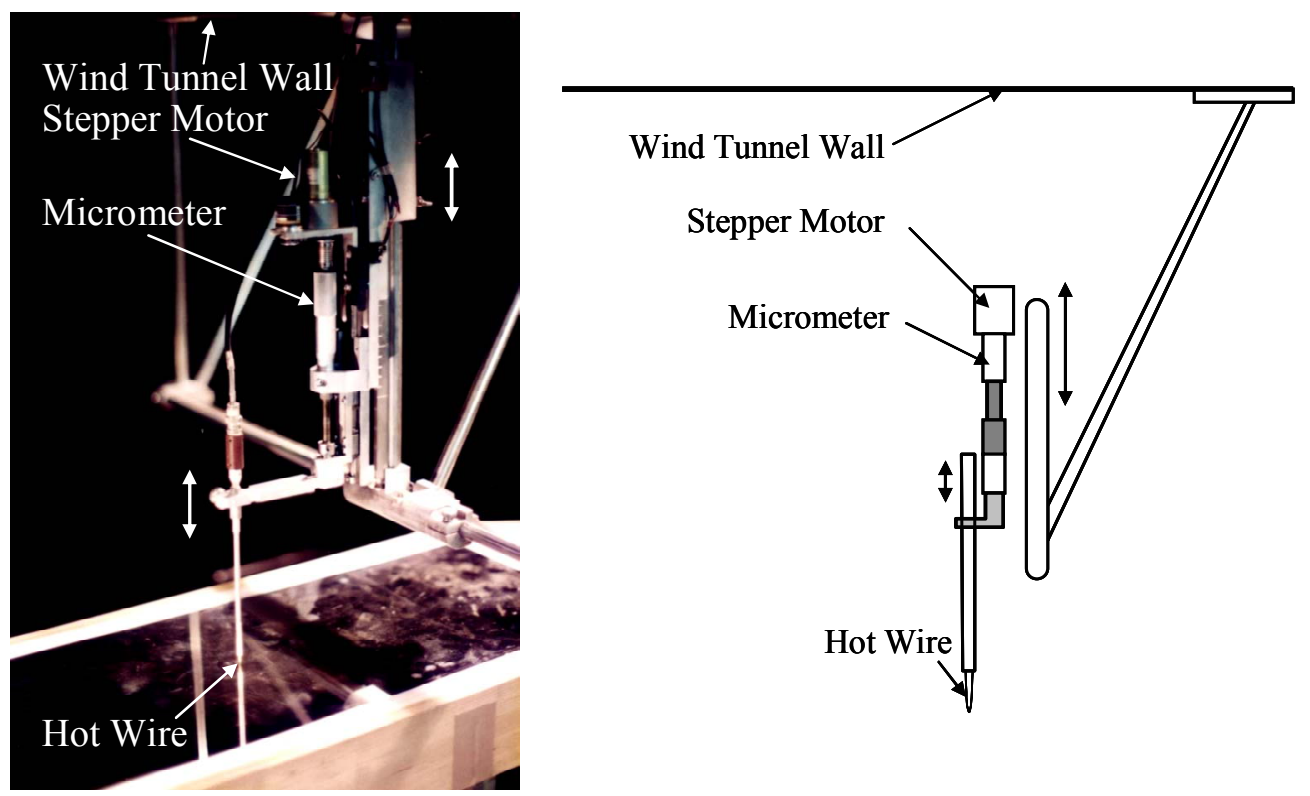

Fig. 5 Traverse unit

Since difference of friction drag among the each wall might be much small, the following two methods were applied. One is to measure total pressure loss in a wake, where $115 \mathrm{~mm}$ downstream from the trailing edge of the basic model (section A). Aerodynamic drag was calculated from the total pressure of the wake on the basis of the following Jones' equation (Fig. 6) ${ }^{(4)}$.

$$
D=2 W \int \sqrt{P_{T A}-P_{S A}}\left(\sqrt{P_{T \infty}-P_{S \infty}}-\sqrt{P_{T A}-P_{S A}}\right) d y
$$

where, $D, P_{T}, P_{S}, \infty$ and $A$ are aerodynamic drag, total pressure, static pressure and subscripts of uniform flow and section A, respectively. And $W$ is the width of the basic model as shown in Fig. 1.

Total pressure in the wake was measured at only the center plane of the basic model under the assumption that the flowfield was approximately 2-dimentional. The location of the total pressure probe was $115 \mathrm{~mm}$ downstream from the trailing edge of the model. 


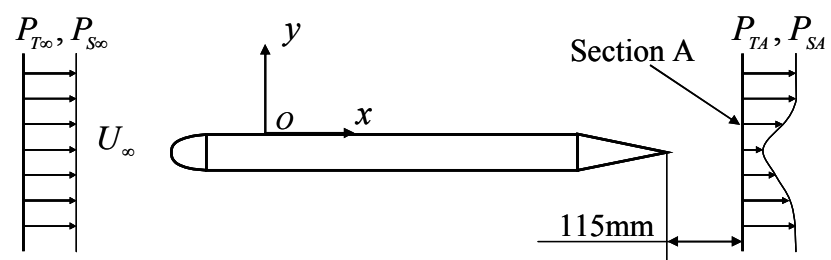

Fig. 6 Drag measurement from total pressure loss

The other method was to calculate a friction drag by using measuring data of a velocity profile at the trailing edge of the compliant or the vibrant walls. In this case, the velocity profile was also measured only at the center plane of the model. Friction drag is estimated from the assumption of uniform distribution of wall shear force $\tau_{w}$ on the compliant or the vibrant wall surface. Wall shear force $\tau_{w}$ is calculated from the velocity gradient near the wall under the assumption that Reynolds stress can be negligible at the viscous sub-layer:

$$
\tau_{w}=\mu \frac{\partial u}{\partial y}
$$

Drag change $\Delta D$ was evaluated by the difference of the drag of the compliant or the vibrant wall from that of the solid wall. $\Delta D$ was non-dimensionalized by the friction drag $D_{0}$ of the solid wall, which was used as the reference value to compare and calculated from the following equation.

$$
D_{0}=\frac{1}{2} \rho U_{\infty}^{2} b \int_{X_{1}}^{X_{2}} C_{f} d x
$$

where, $C_{f}=0.0576\left(U_{\infty} x / v\right)^{-1 / 5}$ is the local skin friction coefficient. And $v, b, X_{1}$ and $X_{2}$ are kinematic viscosity, width of the compliant or the vibrant wall, leading edge and trailing edge location of the compliant or the vibrant wall, respectively.

\section{Results and Discussions}

\subsection{Velocity distribution in the boundary layer}

Velocity profile of the boundary layer at $400 \mathrm{~mm}$ downstream from the leading edge of the compliant and the vibrant wall was measured. A momentum thickness Reynolds number of the static and vibrant wall at this point were 1980 and 2460, respectively. In order to get the detail of the velocity profile, I-type hot wire probe (Kanomax 0251R-T5) was used to measure the flow near the wall. The diameter and length of its sensor are $5 \mu \mathrm{m}$ and $2 \mathrm{~mm}$ respectively. Figure 7 shows the velocity profiles of the compliant and the solid wall, which were normalized by the friction velocity $v^{*}=\sqrt{\tau_{w} / \rho}$. The velocity profiles of the vibrant and the non-vibrant wall are also shown in Fig. 8. The horizontal axes of Figs. 7 and 8 show non-dimensional distance $Y^{+}=y v^{*} / v$ from the wall surface. And the vertical line gives non-dimensional velocity $U^{+}=u / v^{*}$. The velocity profiles of the compliant and the vibrant wall are slightly larger than that of the static wall at the higher area than about $Y^{+} \approx$ 12. From these results, it is considered that some kinds of change in the turbulent flow near the surface might be occurred by vibration.

To calculate the friction velocity $v^{*}$, the wall shear force $\tau_{w}$ was required. It was estimated from the extrapolated velocity gradient from the measurement point nearest to the wall, because it was difficult to measure the wall shear force directly. The accuracy of the estimated wall shear force $\tau_{w}$ was verified using the Clauser chart. On the Clauser chart, the velocity profiles calculated from the $\log$ law $U^{+}=(1 / \kappa) \ln Y^{+}+C \quad(1 / \kappa=2.44, C=5.0)$ against various skin friction coefficient $C_{f}$ values are described as shown in Fig. ${ }^{(5)}$. The plotted data on Fig. 9 are the experimentally measured velocity distribution on the static wall. The skin friction coefficient is determined from Fig. 9, and the wall shear force is calculated with the following equation: 


$$
\tau_{w}=C_{f} \frac{1}{2} \rho U^{2}
$$

As the difference between the wall shear force $\tau_{w}$ calculated from the skin friction coefficient and the estimated $\tau_{w}$ from the slope of the velocity distribution was within $5 \%$, its accuracy was thought to be enough. Therefore in this research, the estimated wall shear forces from the velocity gradient normal to the wall were used.

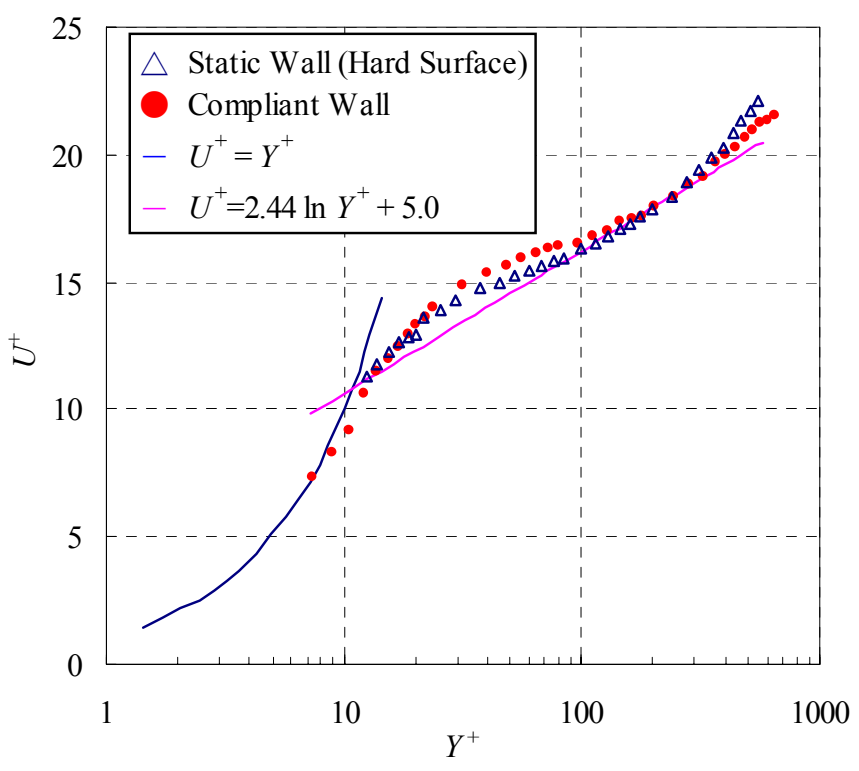

Fig. 7 Velocity profile of the boundary layer on the compliant wall

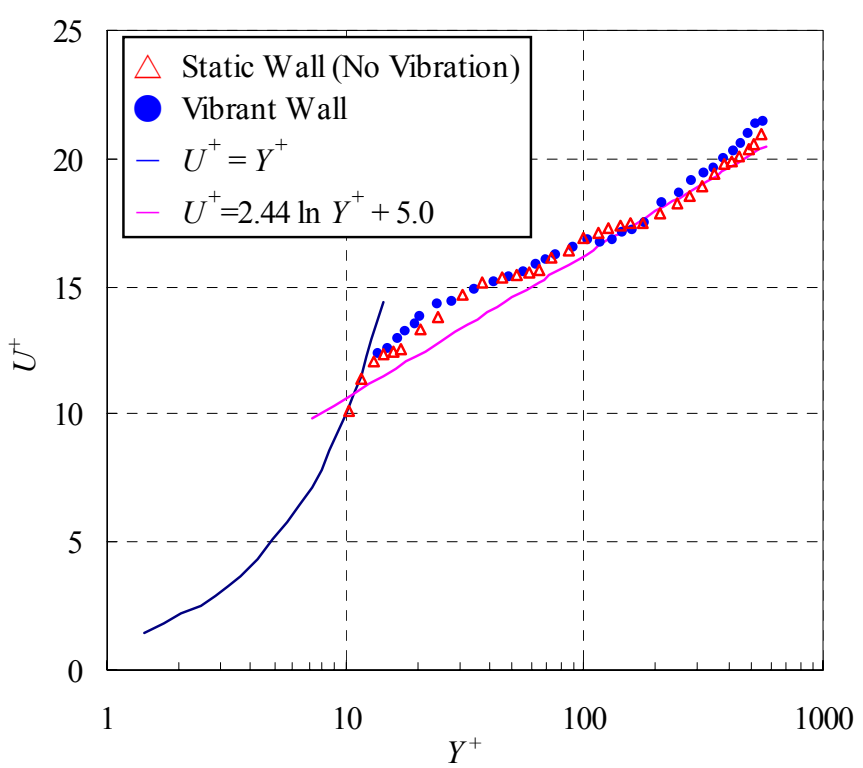

Fig. 8 Velocity profile of the boundary layer on the vibrant wall $\left(f / f_{p}=0.42, a / a_{0}=0.90\right)$ 


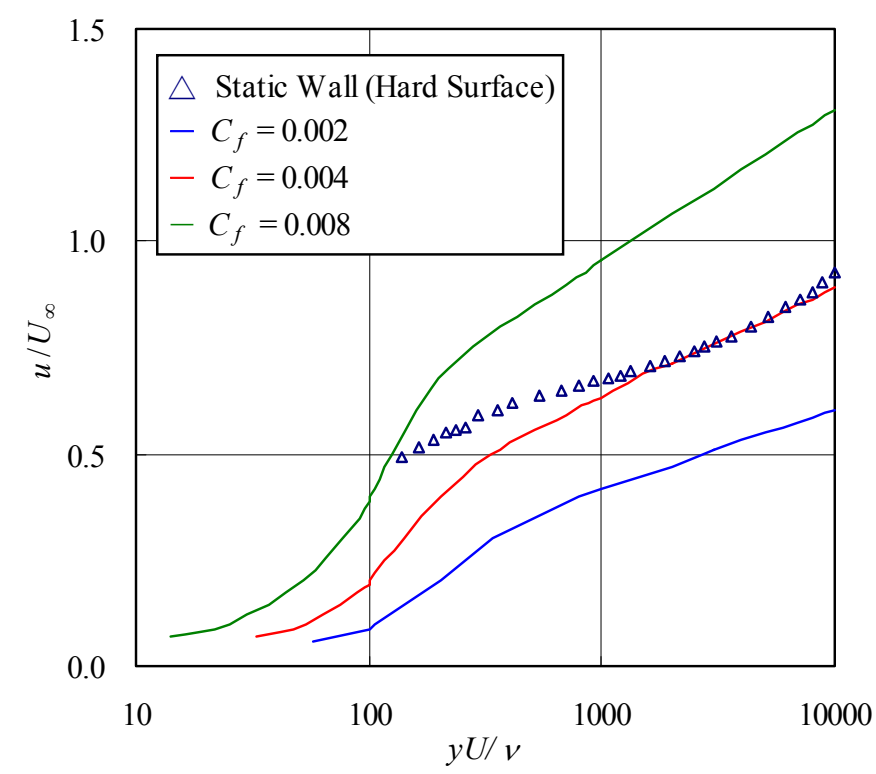

Fig. 9 Estimation of skin friction coefficient from the Clauser chart.

\subsection{Drag measurement of the compliant wall}

Drag difference $\Delta D / D_{0}$ of the compliant wall evaluated by the methods of the 6-component force balance and the total pressure loss is shown in Fig. 10 with changing the width of the uneven gutter. The curve in the figure was obtained by the method of least squares from these results. This result suggested that the aerodynamic drag of the compliant wall has a few percent smaller value than that of the solid wall, and it is recognized that the minimum value is found when the width of the gutter is around $6 \mathrm{~mm}$. On the other hand, there is almost no drag reduction effect at the width of $10 \mathrm{~mm}$.

Passive vibration frequency of the flexible wall was expected to be varied by the width of the uneven gutter. According to this consideration, it was estimated that a certain frequency might relate to the drag reduction effect.

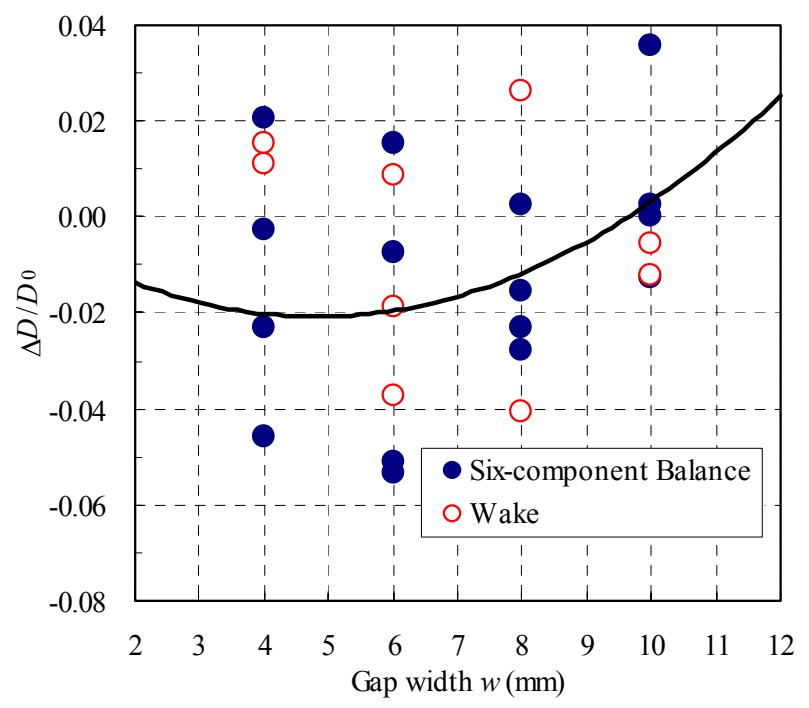

Fig. 10 Drag difference of a compliant wall 


\subsection{Drag measurement of the vibrant wall}

The relation between drag change $\Delta D / D_{0}$ and excitation frequency $f$ of the vibrant wall is shown in Fig. 11. The circles indicate the results from the total pressure loss of the wall wake, and squares are friction drag. Friction drag is integrated by the uniform shear stress assumption. Shear stress is measured at $Y^{+}=15$ by a split film probe. Here, the excitation frequency $f$ was normalized by the maximum frequency of the energy spectrum $f_{p}^{(6),(7)}$, which is expressed as the following equation ${ }^{(8)}$ :

$$
f_{p}=U_{\infty} / 2 \pi \delta
$$

where, $\delta$ shows $99 \%$ boundary layer thickness at the trailing edge of the wall, and it is expressed as follows:

$$
\delta=0.37\left(v / U_{\infty} X\right)^{1 / 5} X
$$

where, $X$ is the distance from the wall leading edge of the model.

In Fig. 11, each result shows the data from individual amplitude of the vibration. Obviously the aerodynamic drag measured by the total pressure loss of the wake (circle) indicates larger differences at every excitation frequency compared with those based on the velocities measured by the split film probe (square). The reason for this can be considered that the wake flow was affected by the 3-dimensional vortex at the trailing edge slope of the test model or insufficient response of the pressure measurement caused by the length of the pressure tube. Furthermore, it is also considered the results from the total pressure loss involve not only friction drag but also pressure drag. Anyway, drag measured by the total pressure loss and by the split film probe show qualitatively same tendency, the drag reduction effect by the vibrant wall seems to be caused by the reduction of friction drag. The solid line shown in Fig. 11 was derived from the friction drag by the least squares method. Amount of drag reduction increases with the increment of excitation frequency up to $f / f_{p}$ of 0.45 , and it takes the maximum reduction value at $f / f_{p}=0.4 \sim 0.5$. Then, the effect decreases as excitation frequency increases. In $f \mid f_{p}$ over $0.8 \sim 0.9$, the drag of the vibrant wall becomes greater than that of the solid wall. This tendency of the drag reduction effect is similar to that of the compliant wall.

Change of aerodynamic drag $\Delta D / D_{0}$ along amplitude of vibration $a$ is shown in Fig.

12. Amplitude $a$ was normalized by $a_{0}\left(=100 v / U_{\infty}\right)$, which is known as Schlichting's surface roughness ${ }^{(4),(6)}$. The relation between the amount of the drag reduction and the amplitude also shows same tendency as the result of Fig. 11. As the vibration amplitude becomes larger, the drag reduction effect increases, and it has the minimum drag at about $a / a_{0}=0.9$. Since the deformation of the wall acts as surface roughness at the condition of $a / a_{0}>1$, drag reduction effect decreases, that is, drag increases conversely. In this study, it is found that there is no drag reduction effect and drag becomes larger in the range of $a / a_{0}>$ 1.8 .

From these results, the optimum frequency and the amplitude of the vibration are $f \mid f_{p}=$ $0.4 \sim 0.5$ and $a / a_{0}=0.9$, respectively. 


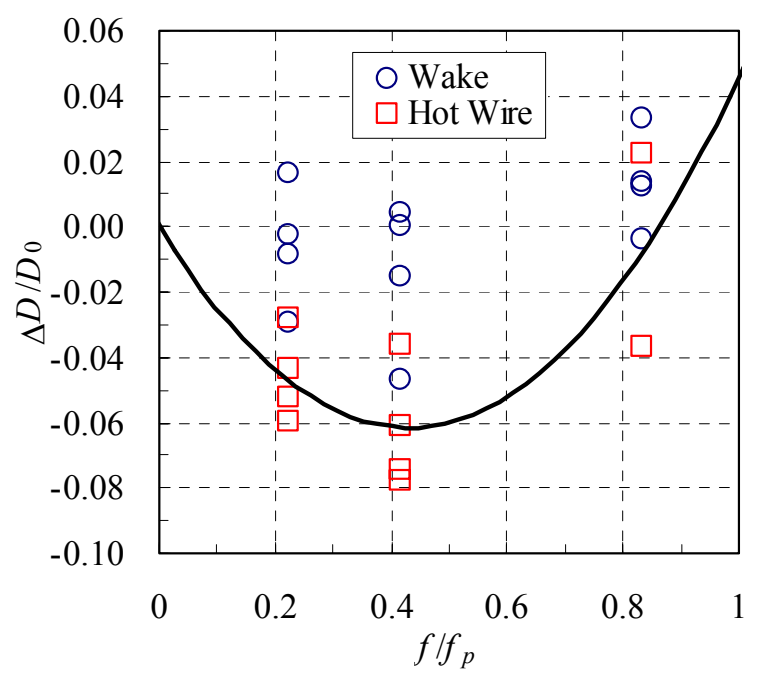

Fig. 11 Drag difference by excitation frequency

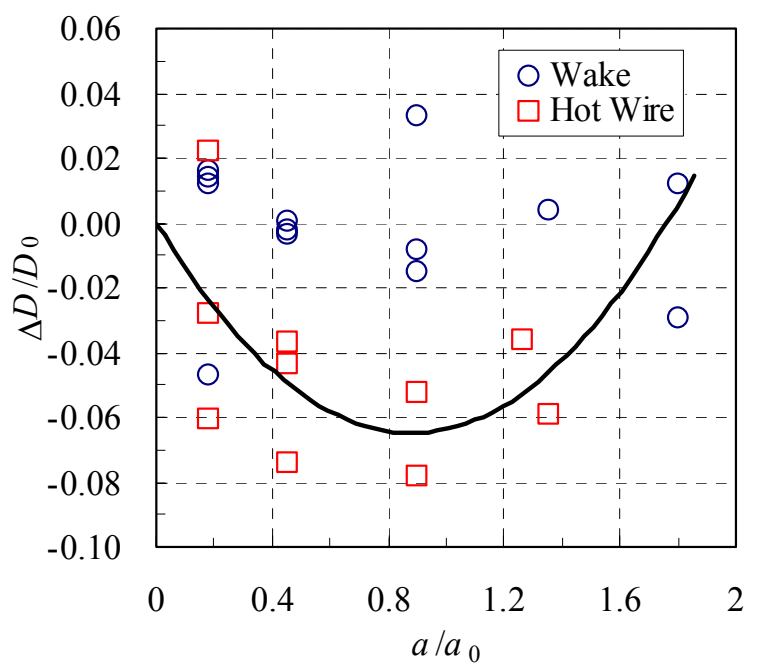

Fig. 12 Drag difference by amplitude of vibration

\subsection{Consideration about velocity profile measured by I-type hot wire probe}

To investigate about the reason for the mechanism of friction drag reduction, velocity distribution and velocity fluctuation of streamwise direction at the trailing edge of the wall were investigated in detail. Velocity distribution $u / U_{\infty}$ is shown in Fig. 13. It is recognized that the flow velocity of the vibrant wall is lower than that of the static wall. And Fig. 14 gives the comparison of the velocity fluctuation $u_{r m s}^{\prime} / U_{\infty}$ between the vibrant wall and the static wall. It shows the velocity fluctuation near the wall is also weakened by vibrating the wall.

According to these results, it was possible to be considered that the decrease of velocity near the wall and the reduction of flow fluctuation energy affect the friction drag reduction. 


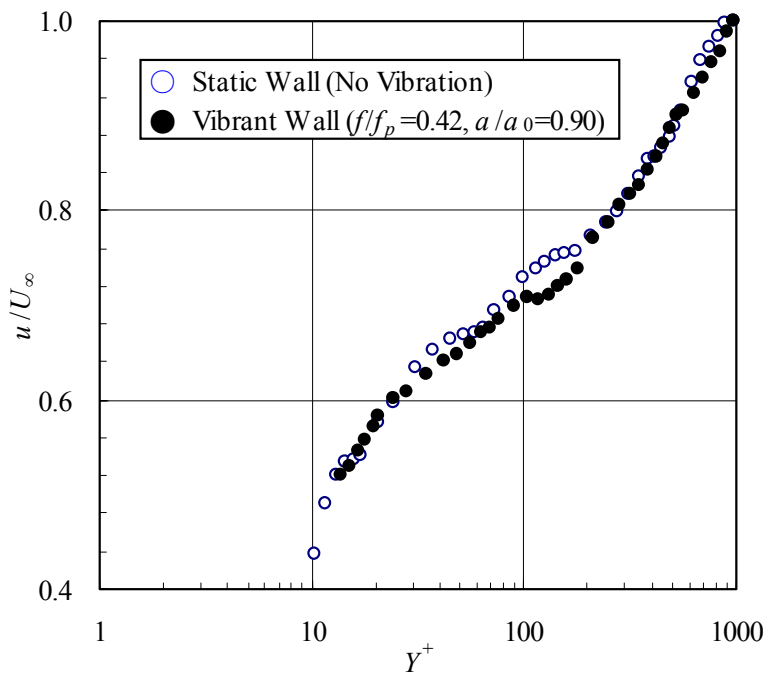

Fig. 13 Velocity distribution in the boundary layer

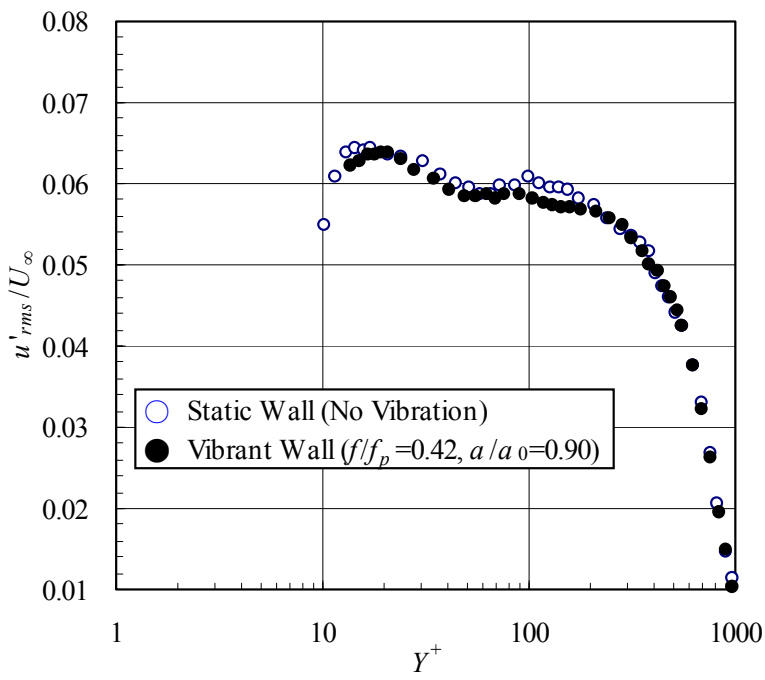

Fig. 14 Velocity fluctuation in the boundary layer

\subsection{Skin friction of the vibrant wall}

Velocity gradient perpendicular to the wall and $x, y$-direction velocity fluctuation $u^{\prime}, v^{\prime}$ at the trailing edge of the vibrant wall $(x=800 \mathrm{~mm})$ were calculated from measurement results of the split film probe. Friction drag was separated into wall shear stress $\mu \partial u / \partial y$ due to velocity gradient and Reynolds stress $-\rho \overline{u^{\prime} v^{\prime}}$. Figure 15 shows the contribution of vibration conditions to the friction drag measured at $Y^{+}=15$. Vibration conditions were 10 patterns constructed by 3 frequencies which were non-dimensionalized by maximum frequency of energy spectrum $\left(f f f_{p}=0.22,0.42,0.63\right)$ and controllable 3 amplitude ranges for each frequencies $\left(a / a_{0}=0.18 \sim 1.36\right)$. In Fig. 15, the far left result shows the case of the solid (no vibration) wall.

The friction drag caused by the velocity gradient $(\mu \partial u / \partial y)$ takes almost constant value at the every vibration conditions. On the other hand, the Reynolds stress $\left(-\rho \overline{u^{\prime} v^{\prime}}\right)$ differs on each vibration condition, and it becomes almost zero at the condition of $f / f_{p}=0.42$ and $a / a_{0}$ $=0.90$. These results show that the drag reduction by the vibrant wall is caused by the decrease of the Reynolds stress due to the mitigation of the velocity fluctuation at the buffer layer, and there is little effect of the shear stress caused by the velocity gradient near the wall. 


\section{Conclusion}

The mechanism of the drag reduction by the compliant and the vibrant wall were investigated experimentally. Conclusions are as follows:

(1) The averaged velocity of the compliant/vibrant wall at the buffer region took slightly larger value than that of the solid wall caused by the vibration of the wall in the turbulent boundary layer.

(2) The drag reduction effect of the compliant wall which has uneven gutters as an inner structure was confirmed. This suggests a passive vibration of a flexible wall relates to decrease the friction drag.

(3) The drag reduction effect of the vibrant wall becomes largest by vibrating the wall with the frequency of $40 \sim 50 \%$ of the maximum energy spectrum frequency and with the amplitude near the Schlichting's surface roughness.

(4) The reduction of the Reynolds stress has large contribution to decrease the friction drag of the vibrating wall. However, the effect of the shear stress caused by the velocity gradient near the wall does not have so much contribution to that.

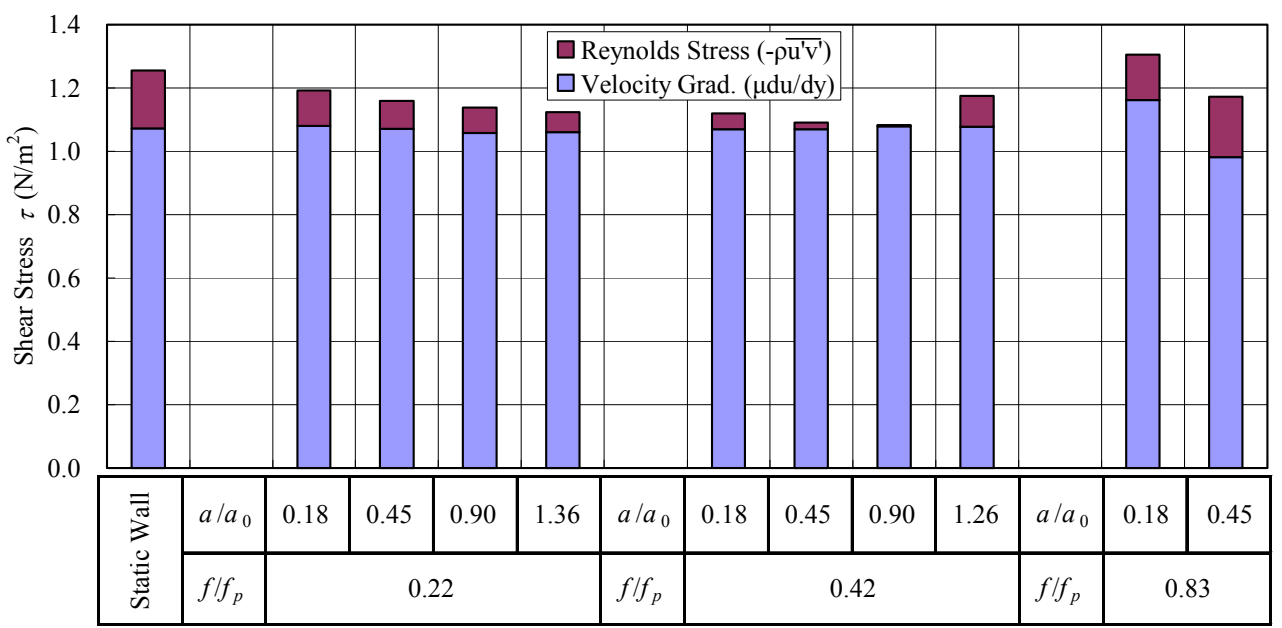

Fig. 15 Contribution to shear stress by each vibration condition

\section{The Main Symbols}

a Amplitude of vibration [mm]

$a_{0} \quad$ Schlichting's surface roughness [mm]

$b \quad$ Width of compliant or vibrant wall [mm]

C Constant [ - ]

$C_{f} \quad$ Local skin friction coefficient [ - ]

$D \quad$ Aerodynamic drag [N]

$f \quad$ Excitation frequency $[\mathrm{Hz}]$

$f_{p} \quad$ Maximum frequency of energy spectrum [Hz]

$L \quad$ Total length of a model [m]

$P_{S} \quad$ Static pressure $[\mathrm{Pa}]$

$P_{T} \quad$ Total pressure $[\mathrm{Pa}]$

$T \quad$ Thickness of a model [mm]

$U \quad$ Free-stream velocity $[\mathrm{m} / \mathrm{s}]$

$u \quad$ Time averaged velocity component along stream direction $[\mathrm{m} / \mathrm{s}]$

$u^{\prime} \quad$ Velocity fluctuation along stream direction $[\mathrm{m} / \mathrm{s}]$

$v^{\prime} \quad$ Velocity fluctuation normal to a wall [m/s]

$v^{*} \quad$ Friction velocity $[\mathrm{m} / \mathrm{s}]$ 
$W \quad$ Width of a model [mm]

$w \quad$ Gap width [mm]

$X \quad$ Distance from model leading edge [mm]

$x \quad$ Coordinate in stream direction [mm]

$y \quad$ Coordinate in normal to a wall [mm]

$\delta \quad$ Boundary layer thickness [mm]

$\kappa \quad$ Von Kármán constant [ - ]

$\mu \quad$ Viscosity [ $\left.\mathrm{kg} \mathrm{s} / \mathrm{m}^{2}\right]$

$v \quad$ Kinematic viscosity $\left[\mathrm{m}^{2} / \mathrm{s}\right]$

$\rho \quad$ Density $\left[\mathrm{kg} / \mathrm{m}^{3}\right]$

$\tau \quad$ Shear stress $\left[\mathrm{N} / \mathrm{m}^{2}\right]$

(Subscripts)

$w \quad$ Wall value

1 Leading edge of a compliant or vibrant wall

2 Trailing edge of a compliant or vibrant wall

$A$ Section located at $115 \mathrm{~mm}$ downstream from trailing edge of a basic model

$\infty \quad$ Uniform flow

\section{References}

(1) Tamano, S., Itoh, S., Matsuda, T., Yokota, K., Drag Reduction in Turbulent Boundary Layer over Flexible Sheet due to Spanwise Traveling Wave Motion, J. Jpn. Soc. Mech. Eng., Ser. B, Vol. 75, No. 757 (2009) pp. 1798-1806 (in Japanese).

(2) Lee, I., Kulik, V. M., Boiko, A. V., Chum, H., Wind-tunnel Measurement of the Drag-reducing Effect of Compliant Coating, World Academy Sci., Eng. Tech., Vol. 55 (2009) pp. 588-592.

(3) Tani, J., Qui, J., Oka, T., Kosugou, K., Murai, M., Boundary Layer Control Using Piezoelectric Actuator, Dynamics and Design Conference 2000, CD-ROM (2009) pp. 1-5 (in Japanese).

(4) Schlichting, H., Boundary Layer Theory, Sixth Edition, McGrow-Hill (1968).

(5) Clauser, F. H., Turbulent Boundary Layers in Adverse Pressure Gradients, J. Aeronaut. Sci., Vol. 21 (1954) pp. 91-108.

(6) Willmarth, W. W., Pressure Fluctuations beneath Turbulent Boundary Layers, Ann. Rev. of Fluid Mech., Vol. 7 (1975) pp. 13-38.

(7) Bull, M. K., Wall Pressure Fluctuations associated with Subsonic Turbulent Boundary Layer Flow, J. Fluid Mech., Vol. 28 (1967) pp. 719-754.

(8) Nakao, S., Possibility of Drag Reduction by Flexible Wall, J. Jpn. Hydraulics Pneumatics Soc., Vol. 15, No. 2 (1984) pp. 116-121 (in Japanese). 